\title{
Torque Ripple Reduction of Brushless DC Motor Using Genetic Algorithm
}

\author{
Tien-Chi Chen ${ }^{1}$, S. H. Shieh ${ }^{1}$, Tsai-Jiun Ren ${ }^{2}$ \\ ${ }^{1}$ Department of Computer and Communication, Kun Shan University \\ No. 195, Kunda Rd., Yong-Kang Dist., Tainan City, Taiwan \\ ${ }^{2}$ Department of Information Engineering, Kun Shan University \\ No. 195, Kunda Rd., Yong-Kang Dist., Tainan City, Taiwan \\ tchichen@mail.ksu.edu.tw; cyrusren@mail.ksu.edu.tw
}

\begin{abstract}
Brushless DC motor torque ripple reduction has been the main issue in servo driving systems in which the speeds of fluctuation, vibration, and acoustic noise should be minimized. Most methods for suppressing torque ripples usually require Fourier series, analysis, finite element analysis or least-mean-square minimization. These methods might lead to errors during complex Fourier series analysis and cost much calculation time. This paper presents a new method to improve torque ripple based on the Genetic Algorithm. The proposed method depends on Genetic Algorithms to search for the Fourier coefficients of three-phase stator currents for the given back-EMF waveforms. These Fourier coefficients can then be used to recompose three-phase optimum current commands for three-phase balanced brushless DC motor driving. The torque ripple must therefore be expected to improve in this way if stator currents are perfectly achieved. The validity and practical applications of the proposed method are verified from experiments using the TMS320F2812 DSP. In the experimental structure, the three-phase optimum current commands and the measured three-phase backEMFs are set up as the tables. They are obtained according to the rotor angle and speed information from the encoder. The experimental results can prove that the proposed method provides a simple and efficient way to obtain three-phase optimum stator currents for the given back-EMF waveforms and the minimum torque ripple will also be acquired.
\end{abstract}

Keywords: Fourier series analysis, genetic algorithm, brushless DC motor, torque ripple

\section{Introduction}

The permanent magnet synchronous motor (PMSM) or brushless DC motor (BLDC motor) are popular in many applications because they can produce acceptable amounts of torque ripple without adding substantial complexity to the motor driving circuitry $[1,2]$. However, the minimum torque ripple is always hard to obtain, because the back-EMF waveforms usually have a non-sinusoidal shape. That will cause large pulsations or ripples in the torque if pure sinusoidal stator current is fed into them. Torque ripple can create undesirable noise and vibration and control inaccuracies in speed applications. Hence, a minimum amount of torque ripple is always required in many applications [3, 4]. Generally, the torque ripple in permanent magnet motors is produced by three physical phenomena: (i) Mutual torque is due to the interactions between the permanent magnet field and the stator currents in the phase windings; (ii) Cogging torque is due to the attraction of the permanent magnet to the salient portions of the stator iron; (iii) Reluctance torque is due to winding self-inductance variations that are a function of the rotor position.

All of these torque ripple sources are greatly affected by the magnetic structure of the permanent magnet motor. For example, cogging torque can be greatly reduced by skewing the magnets relative to the stator slotting; by spacing the stator slots or rotor magnets by one slot. Mutual torque and reluctance torque are also functions of the stator current characteristics. In this view the mutual torque characteristic can be represented as a product of three-phase back-EMFs and the three-phase stator currents. Hence, the back-EMF shape and stator current one will affect the magnitude of the mutual torque ripple if the fed stator current shape is not suitable for the back-EMF shape. The purpose of this paper is to explore the relationship between back-EMFs and stator currents in improving mutual torque ripple [5-7].

The interaction between the three-phase back-EMF waveforms and the three-phase stator current waveforms has been widely studied and analysed by many authors [5-9]. In most of these studies the relationship to the three-phase stator current waveforms, three-phase back-EMF waveforms and torque ripple are analysed using Fourier series and finite 
element analysis. This paper proposes a new method that adopts the Genetic Algorithm [10] to design three-phase optimum current waveforms that produce the minimum torque ripple for a non-sinusoidal back-EMF. Generally, three-phase stator current waveforms can be written as Fourier series expressions. The Genetic Algorithm, widely adopted and studied in various fields [11-13], is applied to search for the most suitable Fourier coefficients for three-phase stator currents after achieving the evolution process included in the reproduction, crossover and mutation operation. The searched Fourier coefficients for the three-phase stator currents can be used to compose three-phase optimum current waveforms which are the most suitable for the given three-phase back-EMF waveforms and provide the minimum torque ripple.

The difference between the proposed method and prior works is that the relationships between three-phase stator current waveforms and three-phase back-EMF waveforms need not be analysed by the complex Fourier series decomposition, finite element analysis, and so on. Many assumptions are not considered here. In practice, some reasons such as manufacturing imperfections, the deterioration of permanent magnets, or unbalanced stator windings such that the assumptions, which are defined in many studies, might lead to errors. The complex Fourier series decomposition might result in errors in the analysis or require much calculation time. Therefore, the proposed method provides a simple and effective way to obtain the three-phase optimum current waveforms and the pure sinusoidal back-EMF waveform is no longer considered. The experimental results prove that the minimum torque ripple can be successfully achieved using the proposed method. The DSP-based BLDC motor drive scheme is adopted to achieve a speed control system with the optimum currents. The feasibility of the proposed method can be examined through presented method.

\section{BLDC Motor Modelling}

For a balanced system, the voltage equations of an inverter-fed three-phase BLDC motor can be expressed as follows:

$$
\left[\begin{array}{l}
v_{a s} \\
v_{b s} \\
v_{c s}
\end{array}\right]=\left[\begin{array}{ccc}
R_{s} & 0 & 0 \\
0 & R_{s} & 0 \\
0 & 0 & R_{s}
\end{array}\right]\left[\begin{array}{l}
i_{a s} \\
i_{b s} \\
i_{c s}
\end{array}\right]+\left[\begin{array}{ccc}
L_{s} & 0 & 0 \\
0 & L_{s} & 0 \\
0 & 0 & L_{s}
\end{array}\right] \frac{d}{d t}\left[\begin{array}{c}
i_{a s} \\
i_{b s} \\
i_{c s}
\end{array}\right]+\left[\begin{array}{c}
e_{a s} \\
e_{b s} \\
e_{c s}
\end{array}\right]
$$

where $v_{a s}, v_{b s}, v_{c s}$ are the applied stator voltages, $i_{a s}, i_{b s}, i_{c s}$ are the applied stator currents, $e_{a s,} e_{b s}, e_{c s}$ are the back-EMF voltage, $R_{s}$ is the stator winding resistance per phase, and $L_{s}$ is the stator winding per phase self-inductance.

The electromagnetic torque can be expressed as

$$
T_{e}=k_{t}\left(i_{a s} e_{a \mathrm{~s}}+i_{b s} e_{b s}+i_{c s} e_{c s}\right)
$$

where $k_{t}$ is torque constant.

The torque, velocity and position can be expressed respectively as follows:

$$
\begin{gathered}
T_{e}=\frac{2}{P} J \frac{d \omega_{r}}{d t}+\frac{2}{P} B \omega_{r}+T_{L} \\
\theta_{r}=\int \omega_{r} d t \\
\omega_{m}=\omega_{r}(2 / P)
\end{gathered}
$$

where $P$ is the number of poles, $\omega_{m}$ is the mechanical angular velocity of the rotor $(\mathrm{rad} / \mathrm{s}), \omega_{r}$ is the electrical rotor angular velocity $(\mathrm{rad} / \mathrm{sec}), \theta_{r}$ is the rotor angular displacement. 


\section{Genetic Algorithm Applied to Reduce Torque Ripple}

The goal of the proposed method is to design three-phase optimum current waveforms, which are to produce minimum ripple in the mutual torque. The mutual torque $\left(T_{e}\right)$ is a cross product of the stator current and back-EMF, as shown as:

$$
T_{e}=\frac{P}{\omega_{r}}\left(e_{a} i_{a}+e_{b} i_{b}+e_{c} i_{c}\right)
$$

where $\omega_{r}$ is the rotor speed, $P$ is the number of poles, $e_{a}, e_{b}, e_{c}$ are the three-phase back-EMFs, and $i_{a}, i_{b}, i_{c}$ are the three-phase stator currents.

Generally speaking, the three-phase stator current $\left(i_{a}, i_{b}, i_{c}\right)$ shape can be written as the Fourier series expressions, which are shown as follows:

$$
\begin{gathered}
i_{a}=\sum_{n=1}^{\infty} I_{n} \sin (n \omega t) \\
i_{b}=\sum_{n=1}^{\infty} I_{n} \sin (n \omega t-2 \pi / 3) \\
i_{c}=\sum_{n=1}^{\infty} I_{n} \sin (n \omega t+2 \pi / 3)
\end{gathered}
$$

where $I_{n}$ is Fourier coefficient of the stator current, and $n$ is the harmonic order.

In order to obtain the three-phase optimal current waveforms, the Genetic Algorithm is applied to search for the Fourier coefficient $I_{n}$ of Eqs. (7), (8) and (9). The three-phase optimal currents can be derived taking the acquired Fourier coefficient $I_{n}$ into Eqs. (7), (8) and (9). The acquired optimal current waveforms must be most suited to the given back-EMF waveforms. Moreover, the mutual torque ripple will be sure to be reduced in opposition.

\subsection{Genetic Algorithm}

John Holland's pioneering book provided a general framework for viewing all adaptive systems (whether natural or artificial) and showed how the evolution process can be applied to artificial systems. Any problems in adaptation can generally be formulated in genetic terms. Once formulated in those terms, such problems can often be solved so-called the "Genetic Algorithm". The Genetic Algorithm is the most effectively method to search the optimum solution. It is a search rules based on the "Darwinism" proposed by Darwinian; therefore, people also call it "propagation algorithm". The Genetic Algorithm concept can be applied to search for the Fourier coefficients of the stator current. Ten stator current harmonic components are considered here (the stator current harmonic components are from 2 nd to 11th and the fundamental current size is fixed as 1). The Genetic Algorithm evolution process includes the reproduction, crossover, mutation and termination conditions. The search process will be introduced in detail in the next section.

\subsection{Initial Population}

The initial parental populations are generated randomly and the generated amount is equal to ten sets in each stator current harmonic component. That is, the number of generated 3rd stator current harmonic components will be equal to ten. The others are generated in the same way. It is very important to appropriately select the population size. If the population size is too small the algorithm will converge too quickly during the evolution process and the worse solutions might be acquired, since the population is unable to provide enough information. On the other hand, if the population size is so too large, the algorithm will require too much training time during the evolution process. The algorithm will then have a difficult acquiring a better solution. Therefore, defining ten sets of every stator current harmonic component implies that it will have one hundred data points in the evolution process. These stator current harmonic components will be increased to twenty sets (two hundred data, which is the sum of the crossover and mutation operation) after completing the first cycle evolution process. 
This advantage of this method is that the different Fourier coefficients of the stator current, which can obtain better results or not, will be provided in the evolution process. Hence, the recombination phenomenon in the small region can be avoided. The current harmonic components of the sinusoidal and rectangular currents are also included in the initial population. The obtained stator current harmonic components can retain the current harmonic components of both originals after completing the evolution process. If better solutions have not yet been found the program is terminated. In this way, the next generation can be ensured to not be worse than the last one. The provided data will also be enough to find a better result.

\subsection{Encoding and Decoding}

In order to effectively search for a better solution, all stator current Fourier coefficients are encoded into the binary strings. How many bits are used for a string? The defined bit string length always depends on the desired precision and search range. The precision of every bit string is expressed as 10 bits in this paper, and the desired search range is defined between -2 and 2 . The search range implies the stator current Fourier coefficient size. The stator current Fourier coefficients $\left(A_{k}\right)$ can be encoded as binary strings using the following encode equation.

$$
A_{k}=a+\frac{b-a}{2^{L}-1} \times \mathrm{g}_{k} \quad k=1,2,3 \ldots
$$

where $b$ is the maximum search range and $a$ is the minimum one, $L$ is the bit string length and $g_{k}$ is the encoded bit string, $k$ is the number of bit strings.

When $g_{k}$ is equal to $1111111111 \mathrm{~b}$, the maximum stator current Fourier coefficient $\left(A_{k}\right)$ will be equal to 1 . On the other hand, when $g_{k}$ is equal to $0000000000 \mathrm{~b}$, the minimum one $\left(A_{k}\right)$ will be equal to -1 . If the $A_{k}$ are substituted into Eq. (3.3), the following expression can be obtained.

$$
\begin{aligned}
i_{a} & =\sum_{n=1}^{\infty} I_{n} \sin (n \omega t) \\
& =I_{1} \sin (\omega t)+I_{2} \sin (2 \omega t)+\ldots+I_{11} \sin (11 \omega t) \\
& =\frac{A_{1}}{1} \sin (\omega t)+\frac{A_{2}}{2} \sin (2 \omega t)+\ldots+\frac{A_{11}}{11} \sin (11 \omega t)
\end{aligned}
$$

where $A_{l}$ is fixed as 1 , the others will be searched through the Genetic Algorithm.

After completing the evolution process the searched bit strings, which are expressed as the binary code, must be decoded to the decimal value again.

\subsection{Fitness}

After completing one cycle of the evolution process the next generations must be checked for fitness to determine if the next generation should be kept or given up. The mutual torque ripple is defined by Eq. (11) as the evolution process fitness. During the evolution process if the fitness becomes smaller mutual torque ripple will be acquired and the composed current waveform becomes more suitable for the given back-EMF.

$$
\text { Torque Ripple }=\frac{\frac{\sum_{k=1}^{M}\left|T_{e}(k)-T_{\text {avg }}\right|}{M}}{T_{\text {avg }}} \times 100 \%
$$

where average mutual torque $\left(T_{\text {avg }}\right)$ is equal to $\sum_{k=1}^{M}\left|T_{e}(k)\right| / M, M$ is the sample points. 


\subsection{Reproduction}

The reproduction operation selects two sets of superior stator current Fourier coefficients as parents from the original populations to produce the next generation. The reproduced probability is proportioned to the fitness. This implies that the stator current Fourier coefficients, which provide the lower fitness, have higher reproduced probability in the evolution process. Depending on this method, more superior stator current Fourier coefficients will be acquired and the generated mutual torque ripple will also be smaller. This is what is called Darwinian natural selection and survival.

\subsection{Crossover}

The crossover operation task is swapping parental bits until the next generation population is equal to that of the original. In this way a more suitable generation might be acquired during the evolution process. The crossover operation is conducted by randomly selecting the crossover points from reproduced bit strings. According to the crossover points the two bit strings are separated into many pieces and swapped with the crossover points in these pieces. After this operation two new bit strings, different from the original ones will be obtained. The crossover points must be appropriately defined; otherwise the original bit string characteristics would be destroyed. Three crossover points are used in the evolution process. The foregoing method is repeated until the new generation population is equal to the original population.

\subsection{Mutation}

After the crossover operation, some bits in the bit strings are arbitrarily changed to produce a new generation with improved characteristics. This method is the so-called mutation operation. The main purpose of mutation is to avoid the solution from falling into local optima (that is searching for a better solution within a small range) during the evolution process. The mutation operation involves randomly selecting bits from the bit strings as mutation bits and arbitrarily changing the state of the mutation bits. This signifies that the original state of mutation bit is 1 . After the mutation operation it will become 0 . On the other hand if the original mutation bit is 0 , it will become 1 .

The number of mutation bits must be appropriately defined. If too many mutation bits are defined, the system will search over a very widely space and obtaining the optimum solution will become harder. On the other hand, if the mutation bit is defined too narrow, it will become meaningless. Three mutation bits are defined in the evolution process.

\subsection{Termination Condition}

Stopping the genetic algorithm is determined by setting the termination condition. One hundred generations is defined as the termination condition in the evolution process. This means that the evolution process will be stopped when 100 executions has been reached.

According to the termination setting the optimum stator current Fourier coefficients will be obtained using the Genetic Algorithm reproduction, crossover and mutation operations. A minimum mutual torque ripple can be acquired from the three-phase optimum current waveforms, composed of the searched stator current Fourier coefficients and then multiplied by the given back-EMF waveform.

\section{Experiment Results}

The experimental results include the sinusoidal current and optimum current for the speed control scheme when a speed command is $200 \mathrm{rpm}$ and $1000 \mathrm{rpm}$, respectively. The speed control scheme is shown in Fig. 1. The back-EMF data are measured beforehand and set up as tables in the TMS320F2812 DSP internal memory. This paper assumed that the back-EMF magnitude is linearly proportional to the speed, hence, the back-EMF factor can be expressed as

$\omega_{r}^{*} \times 6.5 / 1000\left(\omega_{r}^{*}\right.$ is speed command). The three-phase optimum current commands and the three-phase back-EMF waveforms are acquired from the three-phase back-EMF tables according to the rotor position. The speed information is acquired from the shaft encoder. The measured three-phase stator currents are compared with the three-phase current commands through the adaptive current controller to determine the turn-on time for the six PWM switching signals within the predetermined bandwidth. The six PWM switching signals will then be delivered to the inverter. The power transistor switching state will be controlled by these PWM signals such that the stator currents successfully track the desired current commands. 


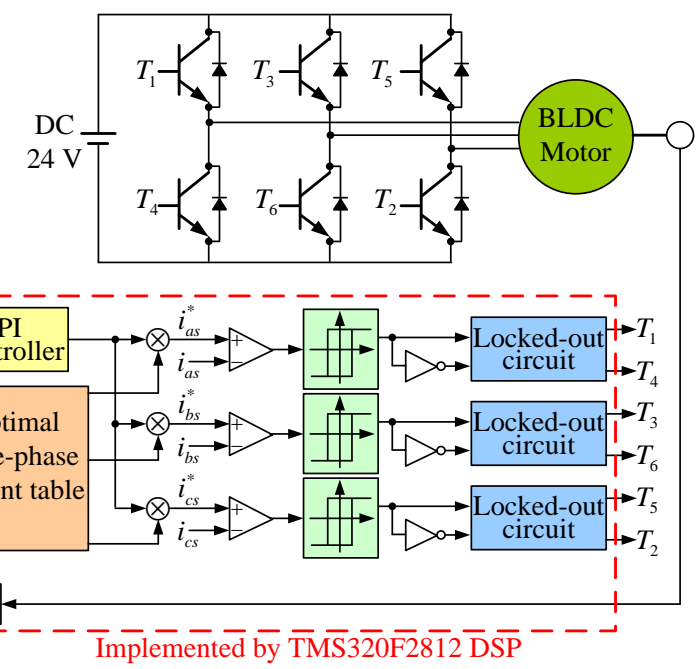

Fig. 1: The speed control scheme.

Two cases bellow will explain the experimental results.

\section{Case I: Speed Command of 200 rpm}

The steady-state stator current, mutual torque transient response and speed response are shown in Figures 2(a)-2(c), respectively, when the speed command is $200 \mathrm{rpm}$ and the sinusoidal current command is fed. The steady-state stator current, mutual torque transient response and speed response are shown in Figures 3(a)-3(c), respectively, when the speed command is $200 \mathrm{rpm}$ and the optimum current command is applied.

According to Figures 2 and 3 the mutual torque ripple for the optimum current scheme is obviously smaller than the sinusoidal current scheme for a low speed command of $200 \mathrm{rpm}$. The mutual torque ripple for the sinusoidal current scheme and optimum current scheme are $13.61 \%$ and $8.76 \%$, respectively. The speed response for the optimum current scheme is also faster than the sinusoidal current scheme.

\section{Case II: Speed Command of $1000 \mathrm{rpm}$}

The steady-state stator current, mutual torque transient response and speed response are shown in Figures 4(a)-4(c), respectively, when the speed command is $1000 \mathrm{rpm}$ and the sinusoidal current command is fed. The steady-state stator current, mutual torque transient response and speed response are shown in Figures 5(a)-5(c), respectively, when the speed command is $1000 \mathrm{rpm}$ and the optimum current command is applied.

From Figures 4 and 5 the mutual torque ripple for the optimum current scheme is obviously smaller than the sinusoidal current scheme for a high-speed command of $1000 \mathrm{rpm}$. The mutual torque ripple for the sinusoidal current scheme and optimum current scheme is $22.87 \%$ and $12.28 \%$, respectively. The speed response for the optimum current scheme is also faster than the sinusoidal current scheme.

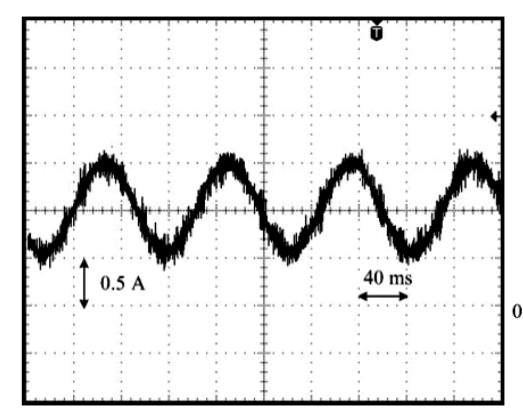

(a)

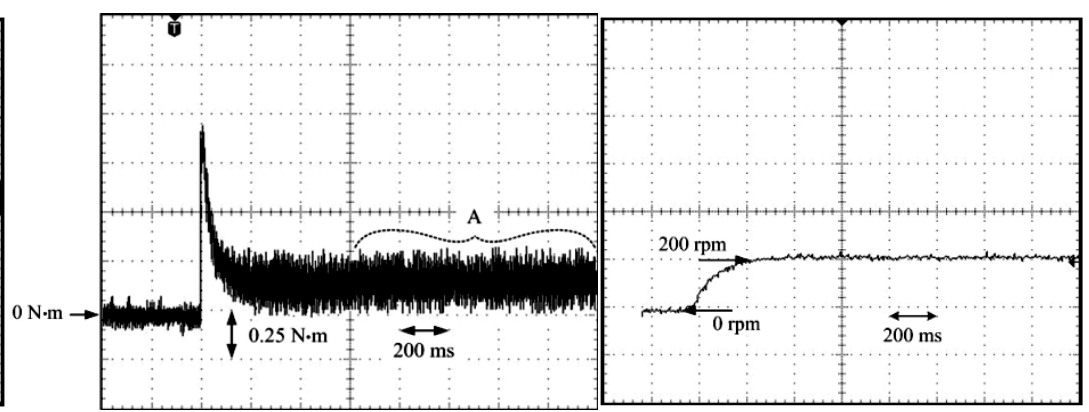

(b) (c)

Fig. 2: The experiment with sinusoidal current command and the speed command of $200 \mathrm{rpm}$. (a) The steady-state stator current, (b) the mutual torque transient response, (c) the speed response. 


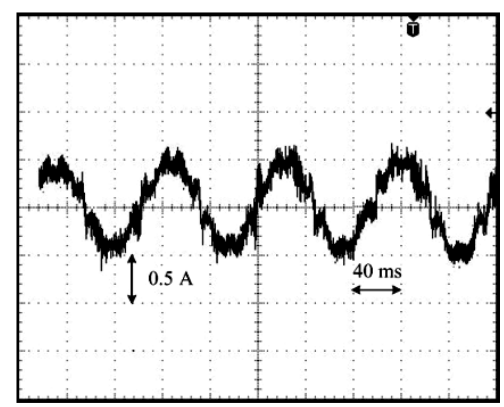

(a)

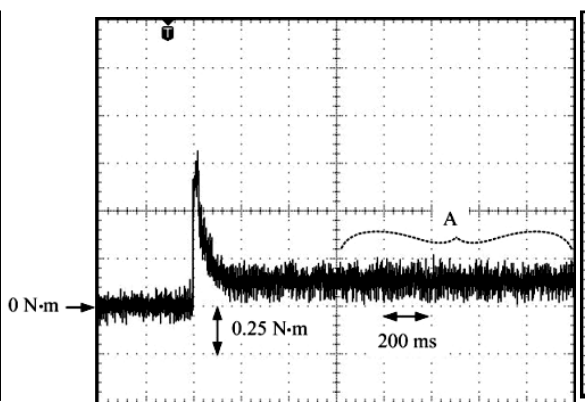

(b)

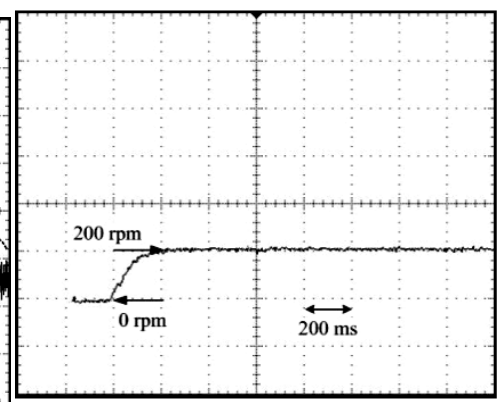

(c)

Fig. 3: The experiment with optimum current command and the speed command of $200 \mathrm{rpm}$. (a) The steady-state stator current, (b) the mutual torque transient response, (c) the speed response.

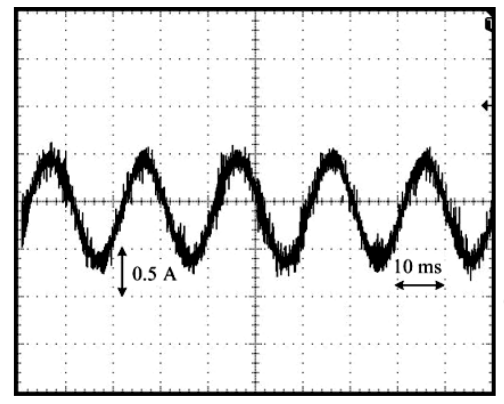

(a)

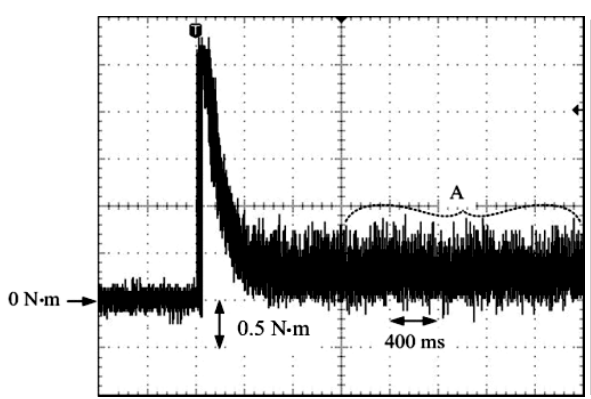

(b)

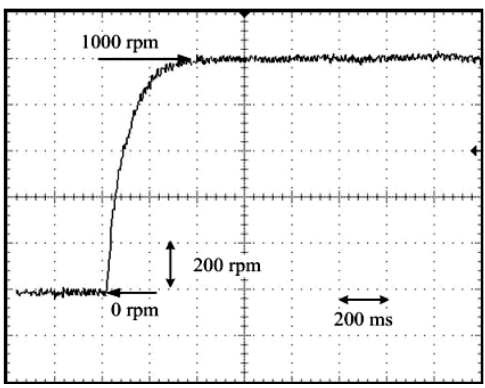

(c)

Fig. 4: The experiment with sinusoidal current command and the speed command of $1000 \mathrm{rpm}$. (a) The steady-state stator current, (b) the mutual torque transient response, (c) the speed response.

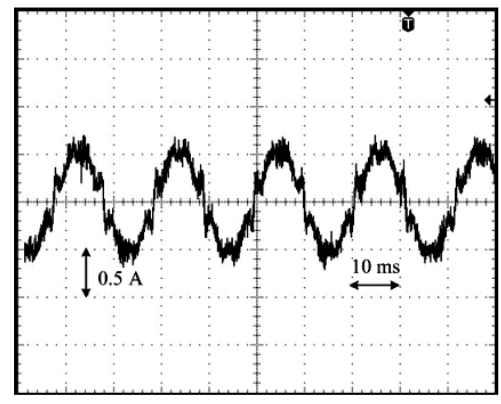

(a)

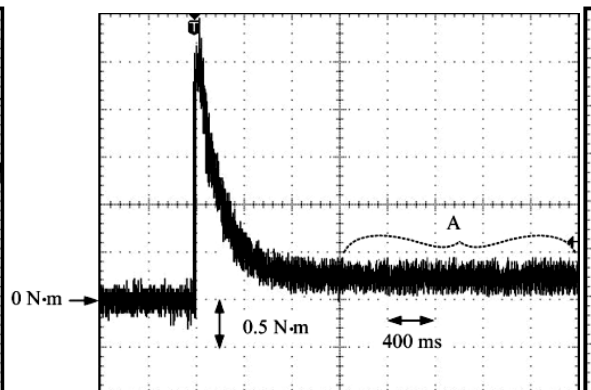

(b)

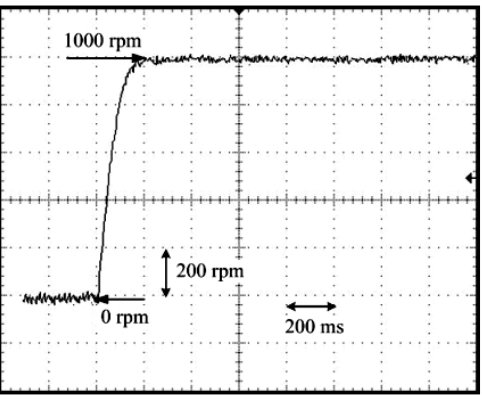

(c)

Fig. 5: The experiment with optimum current command and the speed command of $1000 \mathrm{rpm}$. (a) The steady-state stator current, (b) the mutual torque transient response, (c) the speed response.

\section{Conclusion}

This research developed a new method for designing three-phase optimum stator currents for improving the mutual torque ripple in a three-phase balanced, 6-pole brushless DC motor. For any given back-EMF shape, the optimal stator current Fourier coefficients can be rapidly found using the Genetic Algorithm to compose the three-phase optimum currents. The optimum currents are most suitable for the given back-EMFs and minimum mutual torque ripple will be acquired when these currents are implemented. These experiments were carried out using TMS320F2812 DSP and the mutual torque ripple was evaluated according to the three-phase back-EMFs and measured three-phase stator currents product from the constructed tables according to the rotor angle and speed information.

From experimental results, the stator currents track the desired current commands satisfactorily. The speed response is also faster than the others which feed the sinusoidal stator currents during low or high speeds. The improved mutual torque ripples are expected to become smaller than the others by comparing the mutual torque ripples obtained by feeding the sinusoidal and optimum current commands, respectively. The advantage of the proposed method is that it can be applied to any back-EMFs without the complex Fourier series decomposition or other difficult analysis. Moreover, if the three-phase 
back-EMFs have slightly different shapes from each other, the optimum currents are also found using the proposed method. This proves that the proposed method is a simple and effective way to improve the mutual torque ripple in the brushless DC motor for any given back-EMF shape.

\section{Acknowledgements}

The authors would like to express their appreciation to Ministry of Science and Technology for its support under contract MOST 105-3011-E-168-001.

\section{References}

[1] D. C. Hanselman, Brushless Permanent-Magnet Motor Design. McGraw-Hill International Editions, 1991.

[2] B. K. Bose, Modern Power Electronics and AC Drives. Prentice Hall PTR, 2002.

[3] G. Ranjithkumar and K. N. V. Prasad, "Minimization of Torque Ripple Content for BLDC Motor by Current Controller using MLI," Procedia Engineering, vol. 38, pp. 3113-3121, 2012.

[4] H. M. Hasanien, "Torque ripple minimization of permanent magnet synchronous motor using digital observer controller," Energy Conversion and Management, vol. 51, no. 1, pp. 98-104, 2010.

[5] W. A. Salah, D. Ishak and K. J. Hammadi, "PWM Switching Strategy for Torque Ripple Minimization in BLDC Motor," Journal of Electrical Engineering, vol. 62, no. 3, pp. 1-6, 2011.

[6] S. J. Park and H. W. Park, "A New Approach for Minimum-Torque-Ripple Maximum-Efficiency Control of BLDC Motor," IEEE Trans. on Industrial Electronics, vol. 47, no. 1, pp. 109-114, 2000.

[7] W. Handini, R. Setiabudy and R. Gunaw, "Minimization of Torque Ripple in 24-Slot 16-Pole Inset Permanent Magnet Generator by Edgerounded Magnet Poles and Stator Teeth Notch Techniques," Journal of Theoretical and Applied Information Technology, vol. 93. no.1, pp. 10-16, 2016.

[8] B. Jaganathan, R. Brindha, C. Anuradha and S. Gunasekaran, "A Simple Novel Method of Torque Ripple Minimization in Fuel Cell Based PMSM Drives," ACEEE International Journal on Electrical and Power Engineering, vol. 1, no. 2, pp. 11-15, 2010.

[9] J. Y. Hung and Z. Ding, "Design of Current to Reduce Torque Ripple in Brushless Permanent Magnet Motors," IEE Proceedings Electronic, vol. 140, no. 4, pp. 260-266, 1993.

[10] J. R. Koza, Genetic Programming: on the Programming of Computers by Means of Natural Selection. A Brodford Book, The MIT Press Cambridge, Massachusetts London, England, 1992.

[11] D. S. Weile and E. Michielssen, "The Control of Adaptive Antenna Arrays with Genetic Algorithms Using Dominance and Diploidy," IEEE Trans. on Antennas and Propagation, vol. 49, no. 10, pp. 1424-1433, 2001.

[12] J. A. Vasconcelos, J. A. Ramirez, R.H.C. Takahashi and R.R Saldanha, "Improvements in Genetic Algorithms," IEEE Trans. on Industrial Electronics, vol. 37, no. 5, pp. 3414-3417, 2001.

[13] M. A. S. Masoum, M. Ladjevardi, A. Jafarian; E. F. Fuchs, "Optimal Placement, Replacement and Sizing of Capacitor Banks in Distorted Distribution Networks by Genetic Algorithms," IEEE Trans. on Power Delivery, vol. 19, no. 4, pp. 1794-1801, 2004. 